The Supporting Information:

\title{
Polymerization of oxidized DJ-1 via noncovalent and covalent binding: Significance of disulfide bond formation
}

Mayuka Kobayashi,† Kana Muramatsu,† Takamitsu Haruyama, $\neq \mathbb{I}$ Haruka Uesugi, † Ai Kikuchi, † Hiroki Konno, $\neq$ Noriko Noguchi, $†$ and Yoshiro Saitot\$*

tThe Systems Life Sciences laboratory, Department of Life and Medical Systems, Faculty of Life and Medical Sciences, Doshisha University, Kyoto 610-0394, Japan

¥Nano Life Science Institute (WPI NanoLSI), Kanazawa University, Kanazawa 920-1192, Japan

INara Institute of Science and Technology, Nara 630-0192, Japan

SLaboratory of Molecular Biology and Metabolism, Graduate School of Pharmaceutical Sciences, Tohoku University, Sendai 980-8578, Japan

*Correspondence should be addressed to Yoshiro Saito, Laboratory of Molecular a Biology and Metabolism, Graduate School of Pharmaceutical Sciences, Tohoku University, Aoba-ku, Sendai $980-$ 8578, Japan. E-mail: yoshiro.saito.a8ctohoku.ac.jp. Tel: +81-22795-6870 
Supplemental figure and figure legends of supplemental information

S1

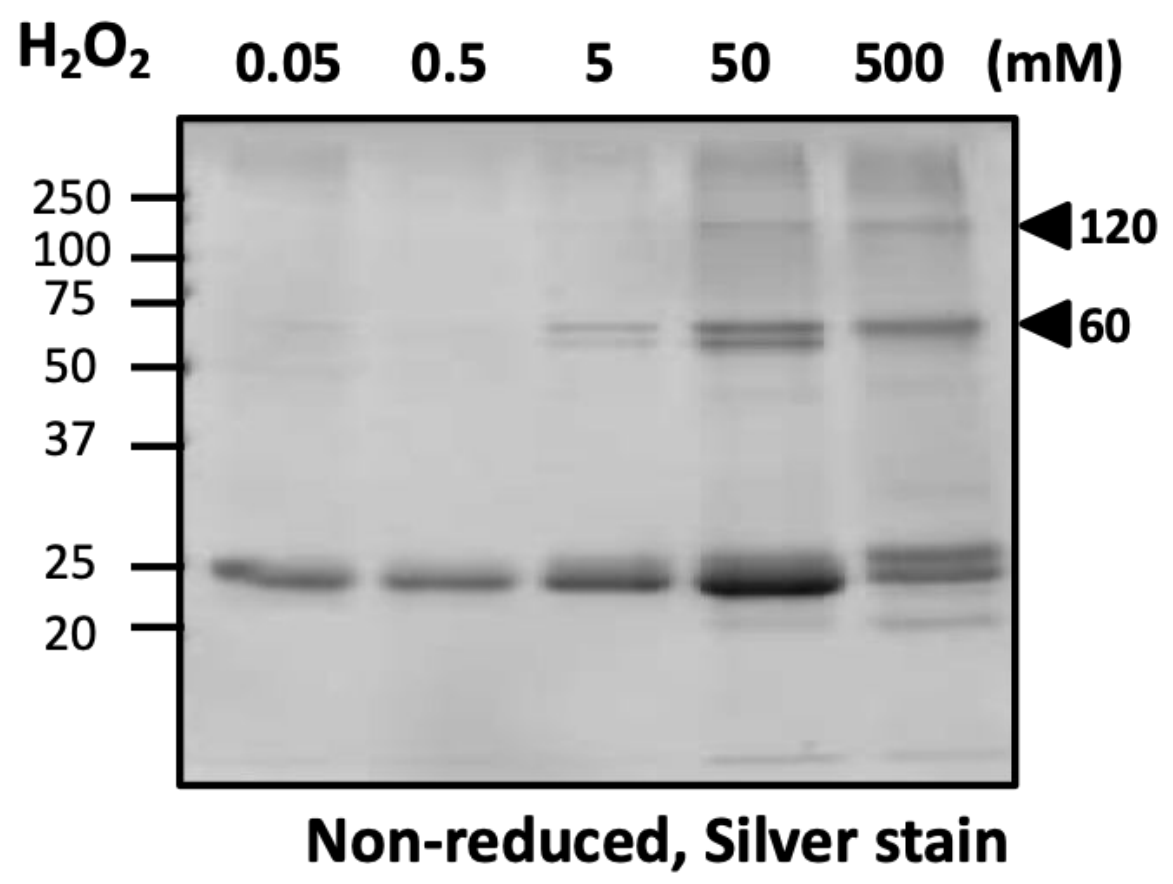

Supplementary Figure S1. The concentration-dependent study of hydrogen peroxide for the formation of oxDJ-1 with high molecular weight. $E$. coli-expressing human DJ-1 were treated with each concentration of hydrogen peroxide at $37^{\circ} \mathrm{C}$ for $15 \mathrm{~min}$, and then oxidized DJ-1 recombinant proteins were purified by Ni-NTA agarose. Purified proteins were subjected to SDS-PAGE under nonreduced condition, and were stained by $\mathrm{CBB}$. 
S2
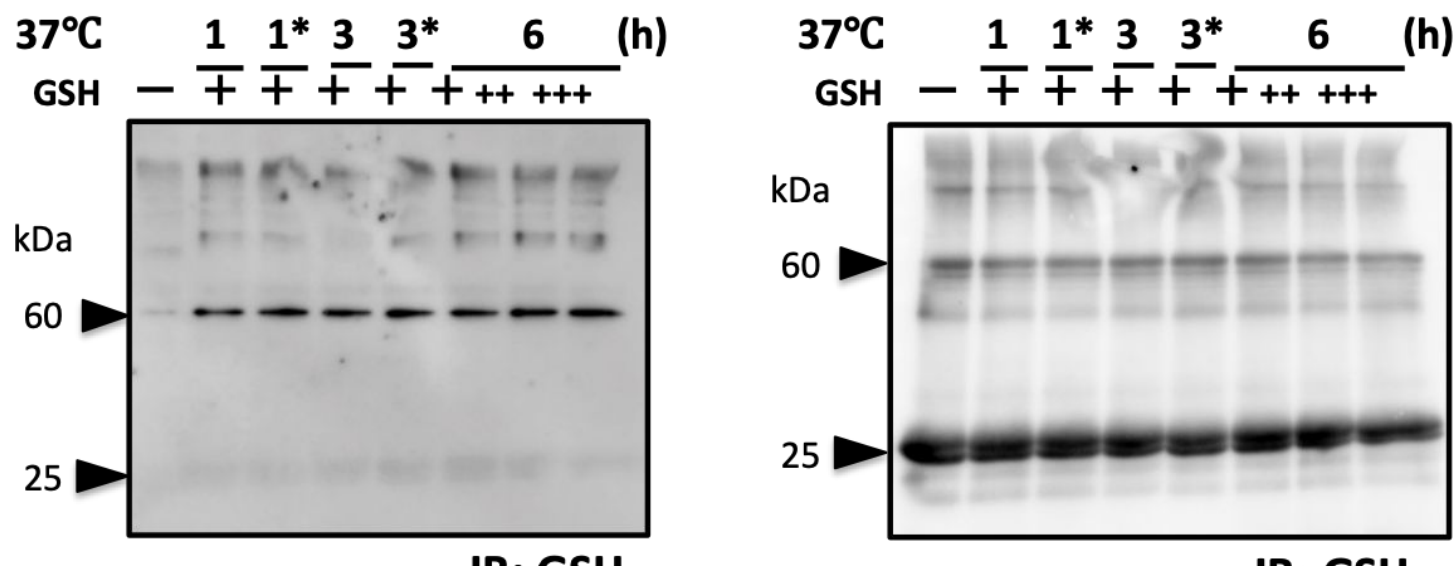

IB: GSH

IB: GSH

Supplementary Figure S2. The time- and concentration-dependent study for the glutathionylation of oxDJ-1. Purified oxDJ-1 protein was incubated with $1 \mathrm{mM}$ glutathione (GSH) for indicated time, and then was subjected to western blotting using anti-GSH and anti-DJ-1 antibody. ${ }^{*}$ oxDJ-1 was incubated with $\mathrm{GSH}$ at $37^{\circ} \mathrm{C}$ for the indicated time and then stored at $4^{\circ} \mathrm{C}$ until $6 \mathrm{~h} .++$ and +++ in the figure indicate 2 and $3 \mathrm{mM} \mathrm{GSH}$, respectively. 


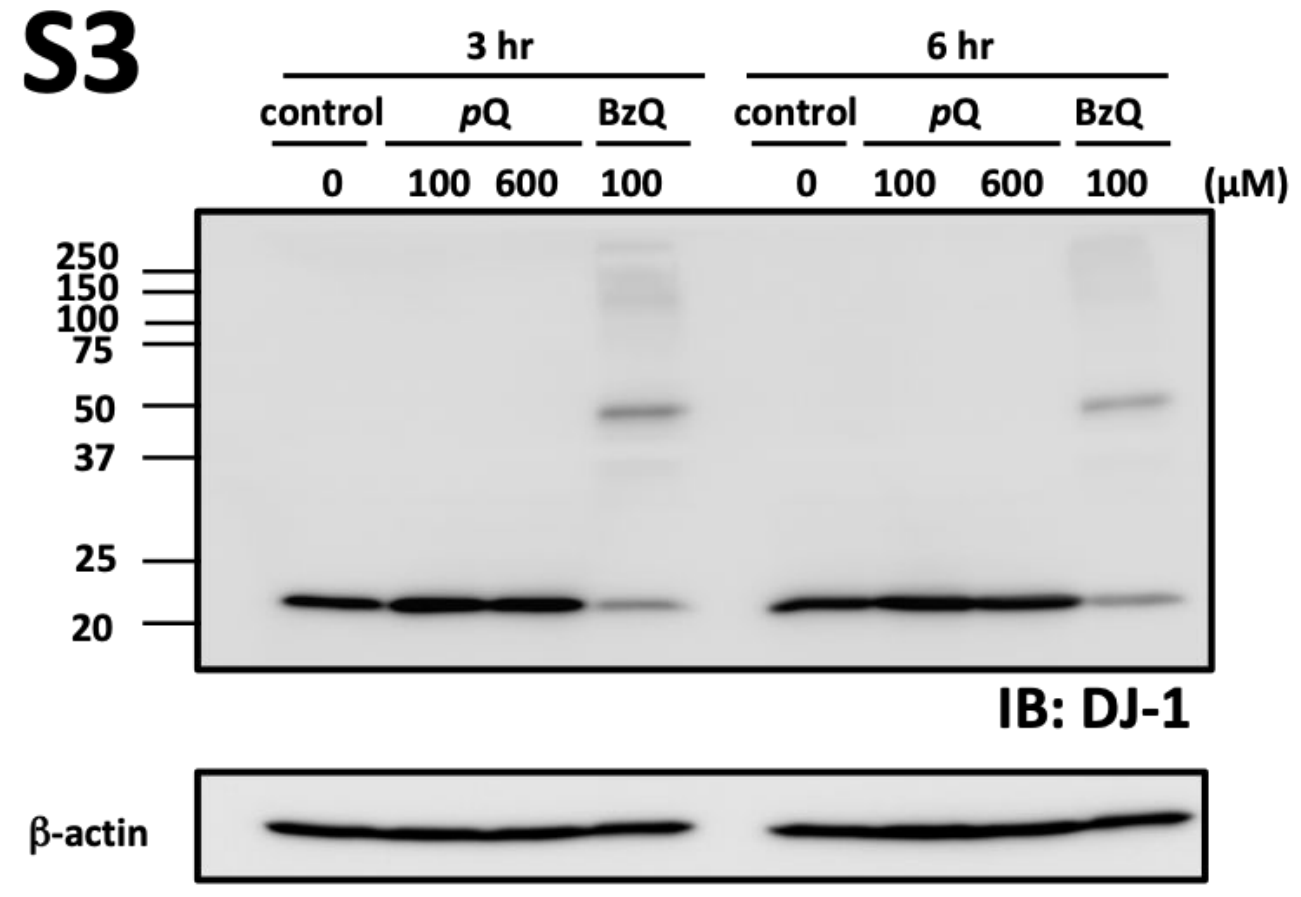

Supplementary Figure S3. The time- and concentration-dependent study for the formation of a covalently bound DJ-1 in SH-SY5Y cells. SH-SY5Y cells were treated with indicated concentration of para-quinone $(p \mathrm{Q})$ and benzoquinone $(\mathrm{BzQ})$ for the indicated time, and then whole cell lysates were subjected to western blotting. 


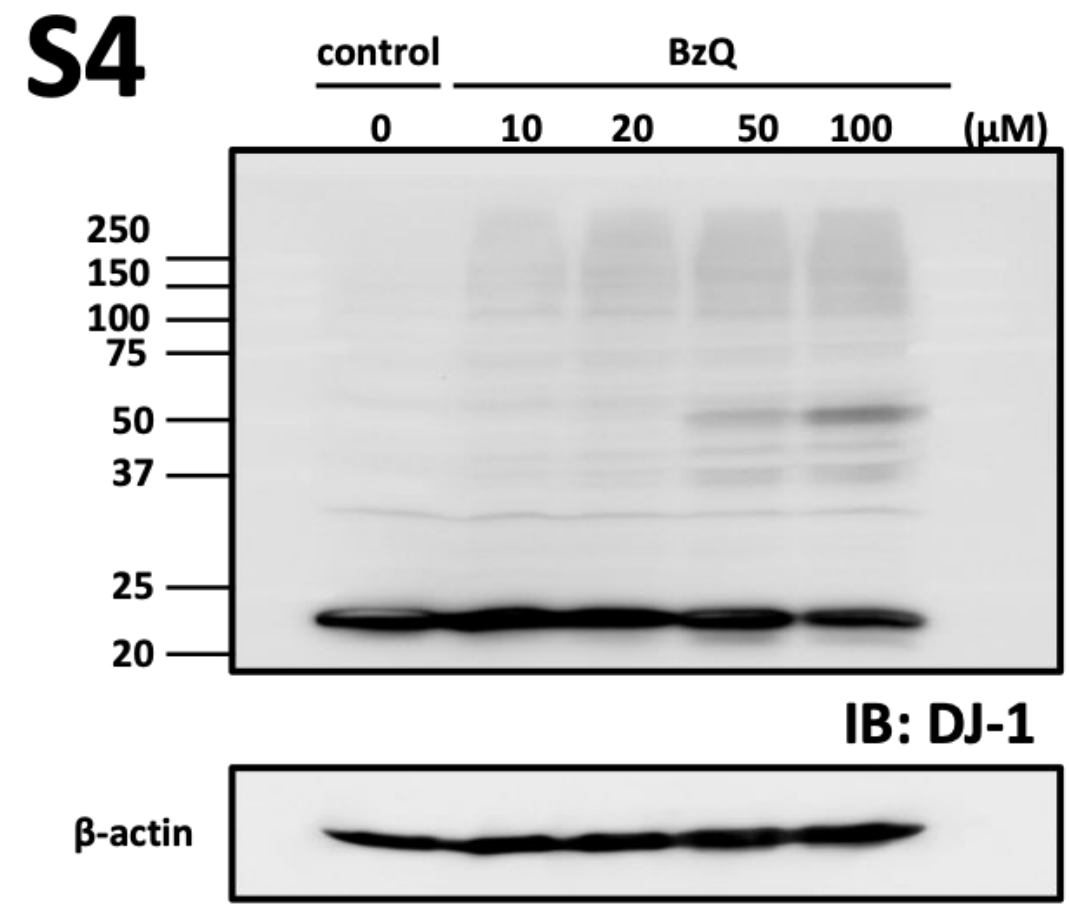

Supplementary Figure S4. The concentration-dependent study for the formation of a covalently bound DJ-1 in SH-SY5Y cells. SH-SY5Y cells were treated with indicated concentration of benzoquinone (BzQ) for $3 \mathrm{~h}$, and then whole cell lysates were subjected to western blotting. 